\section{Antipsychotic drugs and venous thromboembolism}

Sir- The report by Staffan Hägg and colleagues (April 1, p 1155) ${ }^{1}$ about venous thromboembolism and clozapine, leads to the question whether the increased incidence is either: specific for this drug, a general property of antipsychotic drugs, or associated with the underlying condition. After the discovery of the neuroleptic qualities of chlorpromazine and its analogues in the early 1950s, and their widespread application, a higher incidence of venous thromboembolism was described in the German literature between 1953 and 1977. Several studies were of case series, but some contained control groups. A comprehensive study compared two groups of patients between 1953 and $1963 \quad(1172$ schizophrenic or depressive patients on chlorpromazine, amitriptyline, or imipramine ขs 1172 psychiatric patients who did not use neuroleptic or antidepressant medication). The frequency of thromboembolic complications was $2.9 \%$ and $0.6 \%$, respectively. ${ }^{2}$ The report contains multiple references to the contemporary literature, among others a 1959 study by Grahmann and colleagues, who found 11 cases of venous thrombosis among 338 phenothiazine users, versus only one in a non-phenothiazine control group. A historical comparison by Mahmodian, who found a three-fold risk of venous thrombosis in 1958-61, in comparison with 1915-22 in psychiatric and neurology patients was mentioned in a case series report."

We rediscovered this literature, when trying to understand the findings of a study of idiopathic pulmonary emboli deaths. Of 14000 necropsies at the Leiden University Medical Center, Leiden, The Netherlands, from 1970 until mid 1994, we found 27 patients with idiopathic pulmonary embolism for whom sufficient material was present for further investigation; ten of these necropsies were on psychiatric patients. This was an unexpectedly high number. On doing further record searches, we found that five of these patients used neuroleptic drugs; of the non-users of neuroleptic drugs, three had impaired mobility. The necropsy findings and the older literature prompted us to go back to the records of a previous case-control study, ${ }^{5}$ from that study inpatients had been excluded (thus, psychiatric inpatients had been excluded). Nevertheless, four patients of 474 incident cases with a first episode of deep-vein thrombosis had used neuroleptic drugs, compared with none of a similar number of controls.
Venous thrombosis seems to be associated with the use of neuroleptic drugs in psychiatric patients. It cannot be excluded that the findings are an expression of some other underlying factor that could predispose to thrombosis. Nevertheless, we conclude that the association between venous thrombosis and psychiatric illness is worthy of renewed investigation, with an emphasis on the effect of neuroleptic drugs.

\section{René Thomassen}

* Jan $P$ Vandenbroucke, Frits $R$ Rosendaal

Departments of Psychiatry, *Clinicai

Epidemiology, and Thrombosis, and

Haemostasis Research Centre, Leiden University Medical Centre, PO Box 9600, 2300 RC Leiden, Netherlands

(e-mail: vdbroucke@mail.medfac.leidenuniv.nl)

l Hägg S, Spigset O, Söderström TG. Association of venous thromboembolism and clozapine. Lancet 2000; 355: 1155-56.

2 Meier-Ewert $\mathrm{K}$, Baumgart $\mathrm{HH}$,

Friedenberg $P$. Thromboembolische

Komplikationen bei neuro- und

thymoleptischer Behandlung. Dtsch Med Wschr 1967; 47: 2174-78.

3 Scholtz V. Über thromboembolische Komplikationen unter neukroleptischer Medikation. Nervenartz 1967; 38: 174-77.

4 Vandenbroucke JP, Bertina RM Holmes ZR, et al. Factor V Leiden and fatal pulmonary embolism. Thromb Haemostasi 1998; 79: 511-16.

5 Koster T, Rosendaal F, Ronde de H, Briët E, Vandenbroucke J, Bertina $M$. Venous thrombosis due to poor anticoagulant response to activated protein C: Leiden thrombophilia study. Lancet 1993; 342: 1503-06.

Sir-From the twelve cases of clozapine-associated venous thrombosis and thromboembolism Staffan Hägg and colleagues ${ }^{1}$ present, they conclude that the assumed risk of thromboembolism in clozapine-treated patients is at least one per $2000-6000$ Data were retrieved from the database of the Swedish Adverse Reactions Advisory Committee, which is based on spontaneous reporting. This may be a limitation of the study, as was rightly pointed out in the paper. Here, we present data on clozapine-associated thromboembolism in the Arzneimittelsicherheit in der Psychiatrie (AMSP) project. This is a continuing surveillance programme of severe adverse drug reactions (ADRs) in all inpatients of 35 psychiatric hospitals in Germany and Switzerland. ${ }^{2}$ Data are collected by specially instructed drug-monitors, according to a detailed protocol. Results are reviewed in regional and national conferences. Data concerning the screened patient population are compiled in the participating hospitals on two reference days per year. In such a setting, under-reporting of ADRs is less likely and more clinical information of screened and affected patients is available than in the setting described by Hägg and colleagues.

A total of about 103000 inpatients were surveyed from 1993 to 1999 . Of these $13081 \quad(12.7 \%)$ received clozapine, $59637(57.9 \%)$ received other neuroleptics, $30282 \quad(29.4 \%)$ were not treated with neuroleptics. There were five episodes $(0.038 \%)$ episodes of thromboembolism among four patients treated with clozapine. In patients treated with other neuroleptics, there were $17 \quad(0.029 \%)$ cases of thromboembolism, in the group without neuroleptic treatment there were eight $(0 \cdot 026 \%)$ cases. There is no significant difference between these groups.

In our study, the frequency of thromboembolism associated with clozapine treatment is one in 2600 patients. This confirms the work by Hägg and colleagues. However, the incidence of thromboembolism in patients treated with neuroleptics other than clozapine or no neuroleptics was not significantly different from that in the clozapine group. So thromboembolism may not be clozapine-associated after all. Other risk factors must be taken into account, such as reduced motor activity during psychiatric illness. In databases relying on spontaneous reporting, there may be a bias towards reporting $A D R$ in clozapine-treated patients because of the restricted use of that drug. Further research is needed to identify psychiatric patients at risk of developing this potentially fatal complication.

*Jörg Wolstein, Renate Grohmann,

Eckart Rüther, Hanns Hippius

*Department of Psychiatry and Psychotherapy, University of Essen, 45147 Essen; Department of Psychiatry, University of Munich, Munich; and Department of Psychiatry, University of Göttingen, Germany

(e-mail: joerg.wolstein@uniessen.de)

1 Hägg S, Spigset O, Söderström TG. Association of venous thromboembolism and clozapine. Lancet 2000; 355: 1155-56.

2 Grohmann R, Rüther E, Engel RR, Hippius $H$. Assessment of adverse drug reactions in psychiatric inpatients with the AMSP drug safety program: methods and first results for tricyclic antidepressants and SSRI. Pharmacopsychiatry 1999; 32: 21-28.

Sir-Staffan Hägg and colleagues ${ }^{1}$ summarised 12 cases of venous thromboembolism associated with clozapine treatment, that had been submitted to the Swedish Adverse Reactions Advisory Committee. Here we summarise the US experience, based on the spontaneous reporting system of the Food and Drug Administration (FDA). Current labelling of clozapine in the USA mentions the adverse event of pulmonary embolism in the precautions section. Deep vein 
thrombosis (DVT) is mentioned in this section as well as in the postmarketing clinical experience section.

Between February, 1990, when clozapine was first marketed in the USA, and December, 1999, the FDA received 99 unduplicated, domestic reports of venous thromboembolism (83 mentioned pulmonary embolism with or without DVT, and 16 mentioned DVT alone). $63(64 \%)$ reports described deaths as a result of pulmonary embolism; 32 were confirmed by necropsy. Of 36 non-fatal cases, only seven had been documented objectively by such diagnostic techniques as perfusion-ventilation lung scanning and venography. Thus, 39 (39\%) of the 99 reports had objective evidence of pulmonary embolism or DVT. The median age for the 39 individuals was 38 (range $17-70)$ years. $20(51 \%)$ were females. The median daily dose was 400 (range 12.5-900) $\mathrm{mg}$. The median duration of clozapine exposure before diagnosis was 3 months (range 2 days to 6 years). Information on risk factors for pulmonary embolism and DVT varied by report; however 18 of 39 patients were described as obese. The reporting rate for fatal pulmonary embolism in the cohort described above is consistent with that described in the labelling for clozapine in the USA.

Hägg and colleagues concluded that the actual risk of venous thromboembolism in clozapine-treated patients with schizophrenia is probably higher than their estimate, because of underreporting. Under-reporting, however, may not be as substantial in the USA because of the medical attention these patients receive, resulting from the requirement for weekly white-blood-cell counts to be done.

Detection of venous thromboembolism in patients with schizophrenia is clinically challenging. For many reasons, these patients may have difficulty communicating the typical symptoms of DVT and pulmonary embolism. Maintaining a high index of suspicion for the possibility of venous thromboembolism in parients on clozapine may promote earlier diagnosis and appropriate intervention

The views expressed are those of the authors and do not necessarily represent those of, nor imply endorsement from, the FDA or the US Government.

*James F Knudson, Cindy Kortepeter, Greg M Dubitsky, Syed R Ahmad, Min Chen *Division of Neuropharmacological Drug Products (HFD-120) and OPDRA (HFD-430) Food and Drug Administration, Rockville Food and Drug Adm MD 20852, USA

1 Hägg S, Spigset $S$, Soderstrom TG.

Association of venous thromboembolism and clozapine. Lancet 2000; 355: 1155-56.

\section{MRI and the second French case of VCJD}

Sir-Martin Zeidler and colleagues (April 22)' have described a bilaterally increased pulvinar signal on T2weighted magnetic resonance imaging (MRI) of patients from the UK with variant Creutzfeldt-Jakob disease ${ }^{2}$ (vCJD). Here, we report similar routine MRI features in a French patient with vCJD. We also present a description of diffusion-weighted imaging (DWI) in vCJD.

A woman aged 36 years presented with emotional and behavioural changes associated with severe depression. The psychiatric signs worsened despite use of antidepressive drugs, and were followed by progressive dementia, unsteadiness of gait, and cerebellar and pyramidal signs. 7 months after onset, she also developed drowsiness, progressive immobility, unresponsiveness, involuntary movements, and myoclonus. Cerebrospinal fluid examination (including the dosage of 14-3-3 protein) was normal. The electroencephalogram showed bilateral aperiodic slow waves.

Brain MRI done 7 months after symptom onset led to the diagnosis of suspected CJD. Fluid attenuated inversion recovery (FLAIR) images showed bilateral and symmetric high signal changes observed equally in the caudate nuclei, and putamen. Pulvinar high signal accompanied by increased signal in the dorsomedial thalamic nuclei gave the so-called hockey-stick appearance, bilaterally (figure; top). On DWI (not shown), striking hypersignal with decreased values of the apparent diffusion coefficient (ADC) were seen bilaterally in the striatum $(75 \%$ of normal), while only slight hypersignal, with slightly increased $A D C$ values, was observed in the thalami $(116 \%$ of normal).

The patient died 14 months after disease onset and the family gave consent for a necropsy to be done. Neuropathological examination disclosed spongiform changes, diffuse astrogliosis, and numerous florid plaques immunolabelled with monoclonal antibody (figure; bottom). Western-blot analysis showed the presence of type 4 proteinase $\mathrm{K}$ resistant $\mathrm{Pr} \mathrm{P}$, confirming this diagnosis of $\mathrm{VCJD}$.

This constitutes the second French case of vCJD. The patient had never travelled abroad, therefore confirming that continental European and UK vCJD share similar clinical, histopathological, and neuroimaging features. ${ }^{3}$ Our findings confirm those of Zeidler and colleagues, and extend to non-UK patients the potential usefulness of detecting an increased

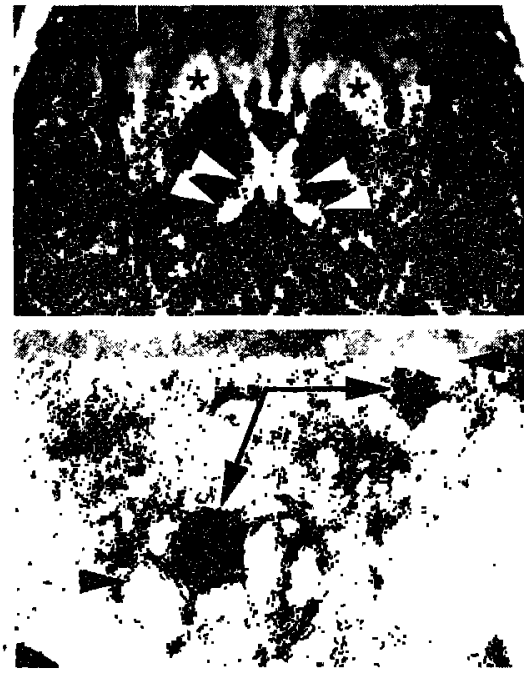

Top: FLAIR MRI showing bilateral increased signal in the caudate nuclei and in the putamen (asterisks), and the pulvinar and the dorsomedial thalamic nuclei (arrowheads)

Bottom: Two florid plaques

immunolabelled with monoclonal antibody directed to PrP

Arrows point to the PrP-positive core arrowheads point to the spongiform changes.

pulvinar signal for the diagnosis of vCJD. The DWI pattern of vCJD was correlated with the known pathological data. We observed slightly increased $A D C$ values in the pulvinar and low $A D C$ values in the striatum. Low $A D C$ values in CJD are presumably a result of spongiform cellular changes, ${ }^{4}$ which, on pathological examination of brains affected by vCJD are seen to predominate in the striatum. Conversely, neuropathological evidence of gliosis and neuronal loss with moderate spongiform changes in the pulvinar are consistent with increased ADC values in the pulvinar.

The relevance of MRI findings in patients with vCJD is highlighted by the results of DWI, which closely parallel the pattern of abnormal tissue seen histologically. The widespread use of MRI in patients with persistent early psychiatric features despite appropriate psychiatric treatment could provide a valuable source of epidemiological data on the emerging epidemic of vCJD, and therefore has public-health implications.

*Catherine Oppenheim,

Jean Philippe Brandel,

Jean-Jacques Hauw, Jean-Philippe Deslys,

Bertrand Fontaine

*Département de Neuroradiologie, Groupe

Hospitalier Pıtié-Salpêtrière, Paris VI University, 75651 Paris, Cedex 13, France; Département de Neuropathologie R Escourolle, Fédération de Neurologie, INSERM U360, Paris; and CEA-

CRSSA Fontenay-aux-Roses Groupe Hospitalter et Faculté de Médecıne Pitié-Salpêtrière, Paris,

France

(e-mail: catherine.oppenheim@psl.ap-hop-paris.fr) 